\title{
A Bibliometric Analysis on Distributed Generation
}

\author{
Peng Peng, Yuying Shao, Xiaoyan Zhu, Xiaodong Lou, Guoqin Yu and Wei Yu \\ State Grid Shanghai Municipal Electric Power Company \\ Shanghai, China \\ E-mail: pengwyw@hotmail.com
}

Keywords: distributed generation, bibliometrics, citation network

Abstract. Distributed Generation can reach multiple functional goals of higher energy efficiency, lower energy cost, higher safety, better environmental protection performance and so on, which is the best way to reflect energy-saving, emission reduction, safety and flexibility. In this paper the development of distributed generation was analyzed from the angle of document metrology. The statistical analysis method had been adopted to study the growth trend, research area and core author of documents and the dynamic evolution of distributed generation was also analyzed by combined co-citation cluster and content words analysis. It was concluded that the research on distributed generation mainly focused on the area of electric power system and its application and the trend of a fluctuant prosperous development was displayed, which was greatly influenced by the government's incentive policy. All these results in this paper provided scholars who research on distributed generation with some reference value.

\section{Introduction}

Bibliometrics is a branch discipline of Library and information science, which uses statistical methods to evaluate the state of a certain field and forecast the future development trends based on various quantitative characters of an extensive literature. Its eventual aim is to obtain the research progress, the current research frontier and the corresponding theoretical basis of a certain subject area. The most frequently used methods are statistics and the literature network visualization [1]. Compared with traditional methods, it can identify important research institutions, scholars and research hot spots more scientifically and accurately and grasp the development trends of scientific research better [2]. In this paper, an extensive literature of distributed generation was combed and profiled by bibliometric and the development characteristics and trends was displayed by adopted the methods of basic statistics and co-citation network map.

\section{Statistics of the Literature of Distributed Generation}

The literature samples in this paper were selected form the CNKI journal database, whose title contains the key words of "distributed generation", in order to select the literature from all fields which is the research emphases or related to the subject of distributed generation closely. The most relevant among all the literature samples is guaranteed by choosing the key word of "distributed generation" in the title instead of the headline. Finally, 746 papers published before September 2016 were selected.

(1) Analysis of the Growth Trend

The quantity and quality of papers is generally considered to be an important indicator to measure the level of discipline development and the output of science and technology, which is also a standard of the achievement and contribution of scientific research. The growth of scientific knowledge are closely related to the growth of literature. The changes in the quantity of literature directly reflects the changes of scientific knowledge [3]. 


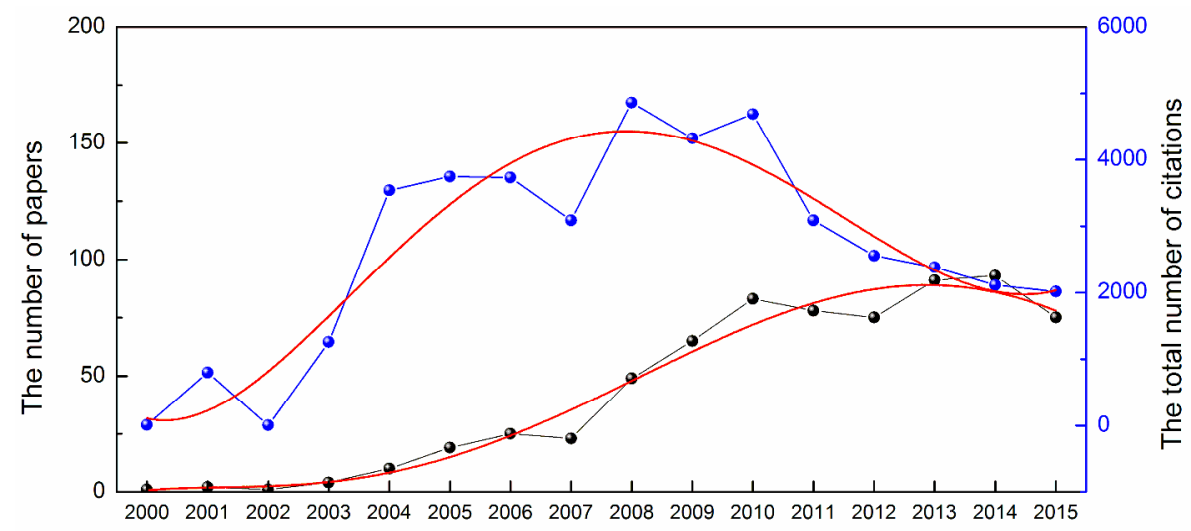

Fig. 1 The total number of literature and citations in each year

The total number of published literature and citations in each year was shown in Fig. 1. From one paper in 2000 to 75 papers in 2015, the quantity of literature had increased by 74 times in 15 years. It indicted that the research field of distributed generation in China burst out after about 2006, and it reached the prosperous periods in 2010, then it entered into the steady development stage. The number of published literature in 2014 achieved the maximum of 93. All the results were consistent with the development of distributed generation in China which was promoted with the major support from the government's incentive policy and that was in accordance with the law of the development of the times. What's more, a rapid growth in the number of citations of distributed generation was displayed in Fig.1. From the 11 citations in 2000 to the peak among 2004 to 2010, the prosperous period of research on distributed generation was achieved. Among the literature samples, the most frequently cited one is a review published in December 2003 [4]. Until now the review had been cited 891 times and the current impact of the main technology of distributed generation on power system was discussed in the paper.

(2) Analysis of Related Research Fields

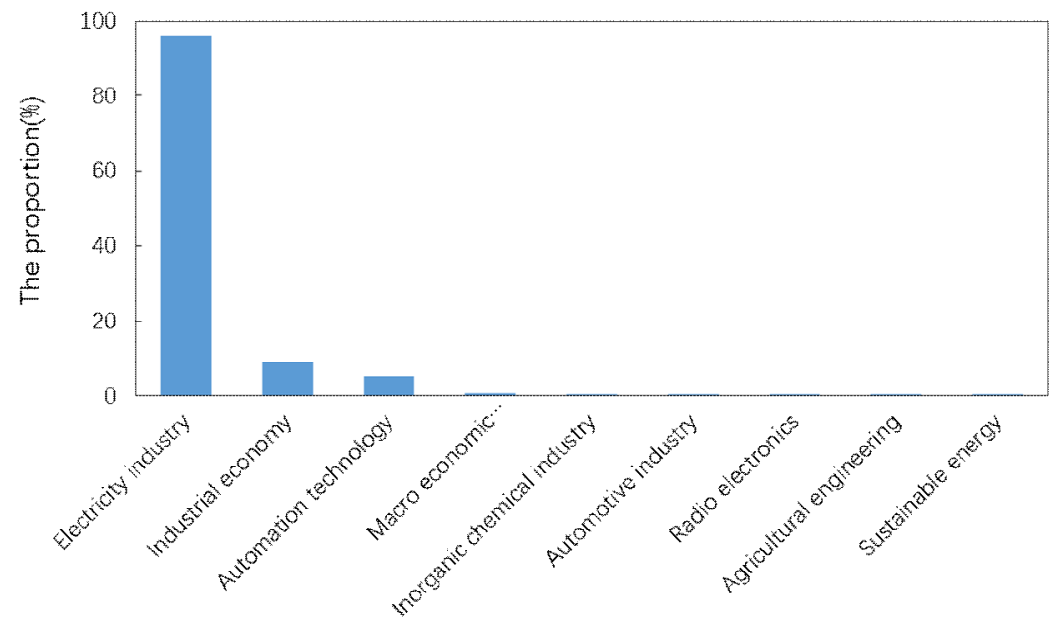

Fig. 2 the major related research fields and their ratio

The current situation and key direction of research on distributed generation can be displayed by statistical analysis of the distribution of related research fields and periodical of literature samples. The major related research fields which were given by the CNKI database were shown in Fig. 2. Among those fields, electricity industry occupied more than $90 \%$ and become the most important research field of distributed generation. In addition, automation technology accounted for $5.36 \%$, while the proportion of sustainable energy was only $0.40 \%$. It indicated that the research field of distributed generation was mainly focused on the application of the power system, while the theoretical basis had not been paid much attention to.

The major periodical in which highly cited papers were published were listed in Table 1. It indicated that literature samples of distributed generation were mainly published in journals of electricity industry, such as Power System Protection and Control, Power System Technology and Automation of Electric Power Systems. The impact factors (IF) of these journals are also higher and all of them are 
included by EI or Chinese core journals. It could be concluded that research on distributed generation was mainly focused on the impact of distributed generation on the grid, grid connected technology and other fields of actual applications.

Table 1 The list of the major periodical in which highly cited papers were published

\begin{tabular}{cccccc}
\hline & No. of citations & No. of papers & Periodical & IF & Source \\
\hline 1 & 3890 & 40 & Power System Protection and Control & 1.895 & Chinese core \\
2 & 3399 & 39 & Power System Technology & 2.389 & EI \\
3 & 5244 & 33 & Automation of Electric Power Systems & 2.671 & EI \\
4 & 211 & 17 & East China Electric Power & 0.420 & Chinese core \\
5 & 264 & 16 & Electrotechnical Application & 0.154 & Chinese core \\
6 & 791 & 16 & Electric Power Automation Equipment & 1.711 & EI \\
7 & 833 & 14 & Proceedings of the CSEE & 2.920 & EI \\
8 & 347 & 13 & Power System and Clean Energy & 0.925 & Chinese core \\
\hline
\end{tabular}

(3) Analysis of Core Authors

The depth and breadth of research on a subject area can be displayed by the statistical analysis of core authors related to the literature samples, as well as the degree of academic exchange [5]. The statistical distribution of the core authors was shown in Table 2. According to Preiss's law, the method of estimating the core author is

$$
M=0.749 \sqrt{N_{\max }} .
$$

$N_{\max }$ stands for the maximum number of papers an authors had published. That is, whose number of papers up to $\mathrm{M}$ can be called core author [6]. The maximum number of papers an authors had published was 17 and $\mathrm{M}$ is 4 in this paper, then authors whose number of papers ranked at the top of 29 could be called core authors. They totally had published 216 papers, accounting for $28.95 \%$ of the total number of paper. It indicated that the research results of distributed generation were mostly concentrated in the smaller range of scholars, and focused on the power system and application. So it needs to be improved on the universal level and the scope of research.

Table 2 The list of core authors

\begin{tabular}{|c|c|c|c|c|}
\hline & Core Authors & No. of authors & No. of papers & The ratios of papers \\
\hline 1 & M. Zeng , C. S. Wang, Y. P. Lu & 3 & $>10$ & $5.63 \%$ \\
\hline 2 & P. Li, D. Q. Gan, B. C. Zou & 3 & 7 9 & $3.22 \%$ \\
\hline 3 & Q. Ai, M. Ding & 2 & 12 & $1.60 \%$ \\
\hline 4 & $\begin{array}{l}\text { J. H. Zhang, Z. Q. Wu, J. Wu, } \\
\text { K. J. Qian, H.Yu, Y. T. Mo, C. D. } \\
\text { Ding, Y. Yuan, H. B. Wu }\end{array}$ & 9 & 45 & $6.03 \%$ \\
\hline 5 & $\begin{array}{l}\text { X. H. Hu, T. Li, X. Zhang, H. P. Cheng, } \\
\text { X. Y. Xia, S. Xue, J. Y. Liu, X. Y. Li, } \\
\text { W. L. Wu, J. Y. Gao, F. Gao, Q. Wang }\end{array}$ & 12 & 48 & $6.43 \%$ \\
\hline & total & 29 & 216 & $28.95 \%$ \\
\hline
\end{tabular}

\section{Co-citation Analysis of Distributed Generation Literature}

The statistical analysis of the distributed generation literature was focused on the general situation of the literature, while the co-citation analysis focused on the contribution and role of the specific literature [7]. In this paper, the citation network of distributed generation was constructed by Citespace. And the key words are clustered to recognize the development trends and dynamic evolution of the distributed generation.

(1) Co citation analysis of scientific research institutions and authors 


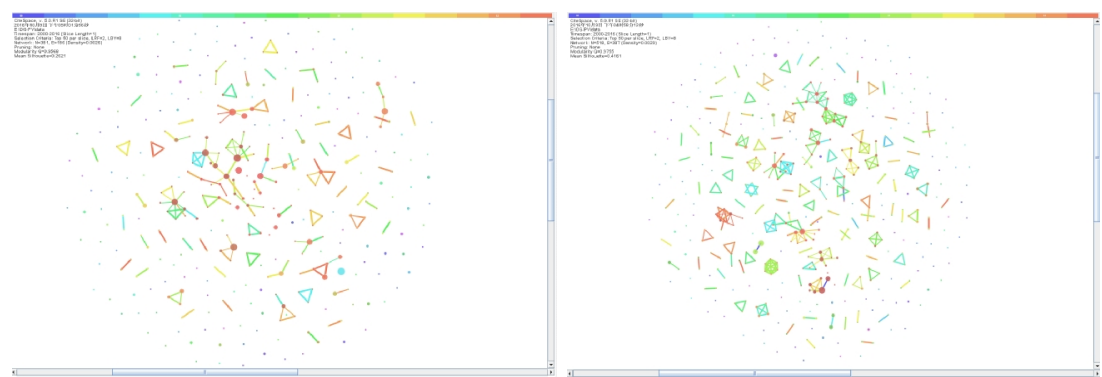

Fig. 3 Citation Network of scientific research institutions (a) and the author (b)

The results of citation network of scientific research institutions and the authors were shown in Fig. 3. The size of nodes represented the frequency of occurrence and the connection between two points represented in the same paper at the same time. It indicated that the relevance of scientific research institutions was poor and there were only a small number of institutions connect with each other. So the academic exchanges between institutions need to be improved. The academic exchanges of scholars also appeared the similar phenomena. These results were consistent with the statistical analysis in the above section. But in the citation network of the authors, there are several centralized points appeared, which indicated that the active academic research exchanges network was only established among the few scholars.

(2) Dynamic evolution analysis of hot spots

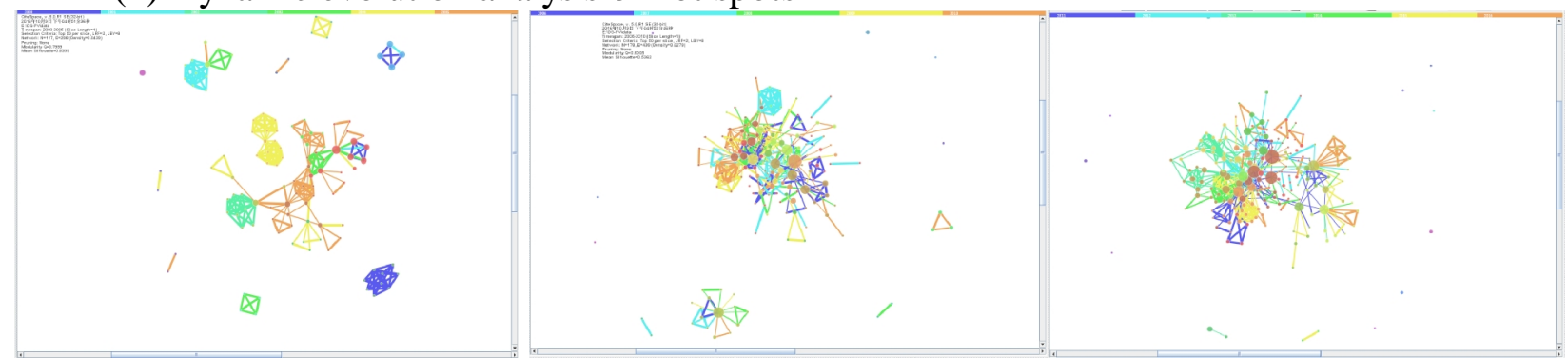

Fig. 4 Dynamic evolution of the citation network of distributed generation

The literature samples were divided into three periods by every 5 years, and the dynamic evolution process of the literature of distributed generation was analyzed by the citation cluster analysis respectively. From the time sequence of the citation network of keywords given in Fig. 4, the development of distributed generation entered into the climax period after 2005 and the prosperous trend was displayed. What's more, The development trends of the research hot spots of distributed generation seemed to be somewhat converged.

\section{Conclusions}

The development and evolutionary process of research on distributed generation were combed and analyzed deeply and systematically by the bibliometrical method in this paper. The basic statistical method was adopted to conduct a comprehensive and preliminary overall analysis of the distributed generation from the growth trend, the breadth of the research fields and the scholars attentiveness. Then the intrinsic connection among literature samples of distributed generation was deeply analyzed and the dynamic evolution of distributed generation was researched through constructing citation network by using Citespace method. Therefore these results in this paper provide a certain reference for scholars and related professionals who pay attention to the development of distributed generation.

\section{Acknowledgements}

This work was financially supported by the technology project of State Grid Shanghai Municipal Electric Power Company (527006160005). 


\section{References}

[1] Y. An, L. Zhang: Library Vol. 5 (2014), p. 63.

[2] T. X. Wen, J. P. Qiu: Information Studies: Theory \& Application Vol. 29 (2006), p. 650.

[3] T. X. Wen, X. Y. Liu: Library and Information Vol. 4 (2008), p. 12.

[4] Y. W. Liang, Z. J. Hu, Y. P. Chen: Power System Technology Vol. 27 (2003), p. 71.

[5] X. R. Li, X. X. Zhang, Z. Y. Li, L. Bo, Q. Zhang, J. X. Zhang, H. Qiao, S. Y. Wang: Systems Engineering-Theory \& Practice Vol. 36 (2016), p. 273.

[6] M. H. Jie, S. B. Jiao, J. X. Guo: Journal of Management Case Studies Vol. 7 (2014), p. 96.

[7] H. T. Wang, F. Q. Wu: Modern Distance Education Vol. 1 (2016), p.3. 